\title{
FINITE $p$-GROUPS IN WHICH EVERY CYCLIC SUBGROUP IS 2-SUBNORMAL
}

\author{
ELIZABETH A. ORMEROD \\ Mathematics Department, Mathematical Sciences Institute, Australian National University, \\ Canberra ACT 0200, Australia \\ e-mail: Elizabeth.Ormerod@anu.edu.au
}

(Received 8 January, 2001; accepted 18 June, 2001)

\begin{abstract}
This paper investigates finite $p$-groups, $p \geq 5$, in which every cyclic subgroup has defect at most two. This class of groups is often denoted by $\mathcal{U}_{2,1}$. The main result is a theorem which characterises these groups by identifying a family of groups in $\mathcal{U}_{2,1}$, and showing that any finite $p$-group in $\mathcal{U}_{2,1}$, with $p \geq 5$, must be a homomorphic image of one of these groups.
\end{abstract}

2000 Mathematics Subject Classification. Primary 20D15. Secondary 20D35.

Introduction. In this paper we characterise finite $p$-groups $(p \geq 5)$ in which every cyclic subgroup is subnormal of defect at most two. Let $\mathcal{U}_{d}$ denote the class of all groups in which every subgroup is subnormal of defect at most $d$, and let $\mathcal{U}_{d, n}$ denote the class of groups in which every $n$-generator subgroup has defect at most $d$. In the case $d=1, \mathcal{U}_{d}$ is the class of Dedekind groups, and $\mathcal{U}_{1,1}=\mathcal{U}_{1}$. For $d=2, \mathcal{U}_{2,1}$ is different from $\mathcal{U}_{2}$ as shown by Ormerod [4] for 3-groups, and Parmeggiani [5] for $p$-groups, $p \geq 3$.

In terms of this notation, we investigate the groups $\mathcal{U}_{2,1}$. Restricted to 2generator $p$-groups, $p$ odd, Mahdavianary [3] has shown that $\mathcal{U}_{2,1}=\mathcal{U}_{2}$, and that any group in $\mathcal{U}_{2,1}$ has nilpotency class at most three. Further he has shown that any group $G \in \mathcal{U}_{2,1}$ if and only if $[v, u, u] \in\langle u\rangle$ for all $u$ and $v$ in $G$. Using this, and the regularity of $p$-groups in $\mathcal{U}_{2,1}$, we prove the following result.

Theorem A. Let $G$ be a finite p-group, $p \geq 5$. Then $G \in \mathcal{U}_{2,1}$ if, and only if, $G$ is a homomorphic image of a group $G_{p}\left(r_{1}, \ldots, r_{n}\right)$, where $1 \leq r_{1} \leq r_{2} \leq \cdots \leq r_{n}$ and

$$
\begin{gathered}
G_{p}\left(r_{1}, \ldots, r_{n}\right)=<a_{1}, b_{1}, \ldots, a_{n}, b_{n}:\left[b_{i}, a_{i}, a_{i}\right]=a_{i}^{3 p^{r_{i}}},\left[b_{i}, a_{i}, b_{i}\right]=b_{i}^{3 p^{r_{i}}},\left[x, a_{i}, b_{i}\right]= \\
{\left[b_{i}, x, a_{i}\right]=x^{p^{r_{i}}},\left[x, a_{i}, a_{i}\right]=\left[x, a_{i}, x\right]=\left[x, b_{i}, b_{i}\right]=\left[x, b_{i}, x\right]=1,} \\
{\left[y, a_{i}, a_{j}\right]=\left[y, a_{j}, a_{i}\right]=\left[y, b_{i}, b_{j}\right]=\left[y, b_{j}, b_{i}\right]=1,\left[y, a_{i}, b_{j}\right]=} \\
{\left[y, b_{j}, a_{i}\right]=1, \gamma_{4}\left(G_{p}\left(r_{1}, \ldots, r_{n}\right)\right)=1>}
\end{gathered}
$$

where $1 \leq i, \quad j \leq n, \quad i \neq j, \quad x \in\left\{a_{1}, b_{1}, \ldots, a_{n}, b_{n}\right\} \backslash\left\{a_{i}, b_{i}\right\}, \quad y \in\left\{a_{1}, b_{1}, \ldots, a_{n}, b_{n}\right\} \backslash$ $\left\{a_{i}, b_{i}, a_{j}, b_{j}\right\}$. 
While writing this paper I was made aware of a similar unpublished result contained in the dissertation of M. Stadelmann [6]. For a group $G$, P. Hall [2] defined an ordered set of elements $p_{1}, \ldots, p_{r}$ of order $\pi_{1}, \ldots, \pi_{r}$ respectively, $\left(\pi_{i} \geq 1\right.$, $i=1, \ldots, r)$ as a uniqueness basis of $G$ if every element $p \in G$ can be expressed uniquely

$$
p=p_{1}^{x_{1}} \ldots p_{r}^{x_{r}}
$$

with $0 \leq x_{i}<\pi_{i}(i=1, \ldots, r)$.

Hall also showed that every regular $p$-group has a uniqueness basis. Stadelmann in his dissertation showed that if $G$ is a $p$-group in $\mathcal{U}_{2,1}$ on $n$ generators $(p>3)$, then there exists a uniqueness basis for $G$, and for each $i, j \in\{1, \ldots, n\}$ there exist integers $\rho_{i j}$ and $r_{i j} \geq 0$ such that $r_{i j}=r_{j i}, \rho_{i j}=-\rho_{j i}, r_{i i}=\rho_{i i}=0,\left(\rho_{i j}, p\right)=1$, for $i \neq j$,

$$
\left[x_{j}, x_{i}, x_{i}\right]=x_{i}^{\rho_{i, j} p^{r_{i j} j}}, \quad \text { for all } i, j \in\{1, \ldots, n\}
$$

and

$$
\left[x_{k}, x_{i} x_{j} x_{k}, x_{i} x_{j} x_{k}\right]=\left(x_{i} x_{j} x_{k}\right)^{\rho_{i k} p^{r_{i k}}+\rho_{j k} p^{r_{j k}}} \quad \text { for all } i, j, k \in\{1, \ldots, n\}
$$

Also, if $G$ is a $p$-group with $\gamma_{4}(G)=1$, and a basis $x_{1}, \ldots, x_{n}$ satisfying these relations, then $G \in \mathcal{U}_{2,1}$.

The work in this paper is quite consistent with Stadelmann's work but gives a much clearer picture of groups in $\mathcal{U}_{2,1}$. Having found such a characterisation of groups in which every cyclic subgroup is 2-subnormal it remains to find more information about $\mathcal{U}_{2}$, the class of groups in which every subgroup is 2-subnormal. As mentioned earlier, $\mathcal{U}_{2}$ is a proper subset of $\mathcal{U}_{2,1}$, so the result in this paper should be helpful. Other subclasses of $\mathcal{U}_{2,1}$ have also been defined, namely $\mathcal{N}$, the class of groups in which every normaliser is normal, and $C$, the class of groups in which the commutator subgroup normalises every subgroup. The class $\mathcal{N}$ is a proper subset of $\mathcal{U}_{2}$ (Parmeggiani [5]) and for $p$-groups, when $p$ is odd, the class $\mathcal{C}$ coincides with $\mathcal{W}_{2}$, the class of groups of Wielandt length two. However, it is not known whether or not the class of $p$-groups ( $p$ odd) in $\mathcal{N}$ coincides with those in $\mathcal{C}$.

Proof of Theorem A. Throughout the rest of the paper assume that $p \geq 5$. The proof of Theorem A is quite long and has many tedious calculations. We aim to keep these to a minimum, subject to providing sufficient evidence of their accuracy. The first step is to prove the sufficiency of Theorem A.

Lemma 1. Let $G_{p}\left(r_{1}, \ldots, r_{n}\right)$ be a group with the presentation given in the statement of Theorem $A$. Then $G_{p}\left(r_{1}, \ldots, r_{n}\right) \in \mathcal{U}_{2,1}$.

Proof. Put $G=G_{p}\left(r_{1}, \ldots, r_{n}\right), r=r_{1}$, and note that $G$ is a regular $p$-group. Since $r \leq r_{2} \leq \cdots \leq r_{n}$, the relations imply that $\gamma_{3}(G)=\left\langle a_{1}^{p^{r}}, b_{1}^{p^{r}}, \ldots, a_{n}^{p^{r}}, b_{n}^{p^{r}}\right\rangle$. It follows that $\gamma_{2}(G)$ has exponent $p^{r}$ and each generator has order $p^{2 r}$. Since $G$ is regular this is enough to show that $G$ has exponent $p^{2 r}$. 
For any $u \in G$ we can write

$$
u=a_{1}^{\alpha_{1}} b_{1}^{\beta_{1}} \ldots a_{n}^{\alpha_{n}} b_{n}^{\beta_{n}} u^{\prime}
$$

where $u^{\prime} \in \gamma_{2}(G)$ and $0 \leq \alpha_{i}, \beta_{i}<p^{r}$. Also $u^{p^{r}}=a_{1}^{\alpha_{1} p^{r}} b_{1}^{\beta_{1} p^{r}} \ldots a_{n}^{\alpha_{n} p^{r}} b_{n}^{\beta_{n} p^{r}}$. To show that $G \in \mathcal{U}_{2,1}$ it will be sufficient to show that $[x, u, u] \in\langle u\rangle$ for all $x \in\left\{a_{1}, b_{1}, \ldots, a_{n}, b_{n}\right\}$. Let $x=a_{i}, 1 \leq i \leq n$. Then

$$
\begin{aligned}
{[x, u, u] } & =\left[a_{i}, a_{1}^{\alpha_{1}} b_{1}^{\beta_{1}} \ldots a_{n}^{\alpha_{n}} b_{n}^{\beta_{n}} u^{\prime}, a_{1}^{\alpha_{1}} b_{1}^{\beta_{1}} \ldots a_{n}^{\alpha_{n}} b_{n}^{\beta_{n}} u^{\prime}\right] \\
& =\left[a_{i}, a_{1}^{\alpha_{1}} b_{1}^{\beta_{1}} \ldots a_{n}^{\alpha_{n}} b_{n}^{\beta_{n}}, a_{1}^{\alpha_{1}} b_{1}^{\beta_{1}} \ldots a_{n}^{\alpha_{n}} b_{n}^{\beta_{n}}\right] \\
& =\prod_{j, k}\left[a_{i}, a_{j}^{\alpha_{j}}, a_{k}^{\alpha_{k}}\right]\left[a_{i}, a_{j}^{\alpha_{j}}, b_{k}^{\beta_{k}}\right]\left[a_{i}, b_{j}^{\beta_{j}}, a_{k}^{\alpha_{k}}\right]\left[a_{i}, b_{j}^{\beta_{j}}, b_{k}^{\beta_{k}}\right] \\
= & \left(\prod_{j \neq i}\left[a_{i}, a_{j}^{\alpha_{j}}, b_{j}^{\beta_{j}}\right]\left[a_{i}, b_{j}^{\beta_{j}}, a_{j}^{\alpha_{j}}\right]\left[a_{i}, a_{j}^{\alpha_{j}}, b_{i}^{\beta_{i}}\right]\left[a_{i}, b_{i}^{\beta_{i}}, a_{j}^{\alpha_{j}}\right]\left[a_{i}, b_{j}^{\beta_{j}}, b_{i}^{\beta_{i}}\right]\left[a_{i}, b_{i}^{\beta_{i}}, b_{j}^{\beta_{j}}\right]\right) \\
& \times\left[a_{i}, b_{i}^{\beta_{i}}, a_{i}^{\alpha_{i}}\right]\left[a_{i}, b_{i}^{\beta_{i}}, b_{i}^{\beta_{i}}\right] \\
= & \left(\prod_{j \neq i} a_{i}^{\alpha_{i} \beta_{j} p^{r_{j}}} a_{i}^{-\alpha_{i} \beta_{j} p_{j}^{r_{j}}} a_{j}^{-\alpha_{j} \beta_{i} p^{r_{i}}} a_{j}^{-2 \alpha_{j} \beta_{i} p^{r_{i}}} b_{j}^{-\beta_{j} \beta_{i} p^{r_{i}}} b_{j}^{-2 \beta_{j} \beta_{i} p^{r_{i}}}\right) \\
= & \times a_{i}^{-3 \alpha_{i} \beta_{i} p^{r_{i}}} b_{j}^{-3 \beta_{i}^{2} p^{r_{i}} .}
\end{aligned}
$$

A similar calculation shows that $\left[b_{i}, u, u\right]=u^{3 \alpha_{i} p^{r_{i}}}$. Hence $G \in \mathcal{U}_{2,1}$.

We now move to the rest of the proof of Theorem A. The proof relies very much on the facts that $G_{p}\left(r_{1}, \ldots, r_{n}\right)$ has nilpotency class three, and that $p \geq 5$, ensuring that $G_{p}\left(r_{1}, \ldots, r_{n}\right)$ is a regular group. The choice of generators is also crucial. Since 2-generator groups in $\mathcal{U}_{2,1}$ belong to $\mathcal{W}_{2}$, the class of groups of Wielandt length two, some properties of these groups are used extensively. When $p$ is an odd prime, if a group $G$ has Wielandt length two, the commutator subgroup $G^{\prime}$ is in the Wielandt subgroup. Elements of the Wielandt subgroup induce power automorphisms, and for regular finite $p$-groups, power automorphisms are universal (see Cooper [1, 5.3.1]). Hence if $w$ is an element of the Wielandt subgroup of a regular finite $p$-group $G$, then there is an integer $n$ such that $[w, g]=g^{n}$ for all $g \in G$. It follows then that if $x$ is an element of maximal order in $G$ and $[w, x]=x^{m}$ for some integer $m$, then $[w, g]=g^{m}$ for all $g \in G$. In particular, if $[w, x]=1$ then $[w, g]=1$ for all $g \in G$. Let $g, h$ be elements of a group $G \in \mathcal{U}_{2,1}$. Since $\langle g, h\rangle$ has Wielandt length two, there are integers $\alpha$ and $r \geq 1$ such that $[g, h, x]=x^{\alpha p^{r}}$ for all $x \in\langle g, h\rangle$. The integer $\alpha$ is not unique, since if $|x|=p^{m}, x^{\alpha p^{r}}=x^{\alpha\left(p^{r}+p^{m}\right)}=x^{\alpha\left(1+p^{m-r}\right) p^{r}}$. However, the integer $r$ remains unchanged. If $[g, h, x] \neq 1$ for all $x \in\langle g, h\rangle$, put

$$
r_{g h}:=\left\{r:[g, h, x]=x^{\alpha p^{r}} \text { for all } x \in\langle g, h\rangle,(\alpha, p)=1\right\} .
$$

If $[g, h, x]=1$ for all $x \in\langle g, h\rangle$, put $r_{g h}:=\infty$. For every $g \in G$, set

$$
\mathcal{R}(g):=\left\{r_{g h}: h \in G\right\} .
$$

Also we use $\Phi(G)$ to denote the Frattini subgroup of $G$. 
Lemma 2. Let $G$ be a group in $\mathcal{U}_{2,1}$ of class 3 . Then

(i) there exists $a \in G$ of maximal order and $x \in G$ such that $[x, a, a] \neq 1$,

(ii) there exists a of maximal order in $G$ and $b \in G \backslash\langle a\rangle \Phi(G)$ such that $[b, a, a] \neq 1$.

Proof. (i) If $[v, u, u]=1$ for all $v, u \in G$, then $G$ has class 2 , contrary to the assumption. So there exist $u$ and $v$ in $G$ such that $[v, u, u] \neq 1$. If $u$ is of maximal order, the proof is complete. If $v$ is of maximal order, $[v, u, u] \neq 1$ implies $[v, u, v] \neq 1$ and again the proof is complete. If neither $u$ nor $v$ is of maximal order, let $a$ be an element of maximal order. Then $|a v|=|a|$ and $[a v, u, u]=[a, u, u][v, u, u]$, where either $[a v, u, u]$ or $[a, u, u]$ is non trivial. If $[a, u, u] \neq 1$, then $[a, u, a] \neq 1$, giving the required result. A similar result follows if $[a v, u, u] \neq 1$.

(ii) From part (i) we can find $x$ and $a$ such that $[x, a, a] \neq 1$. If $x \notin\langle a\rangle \Phi(G)$, there is nothing to prove. If $x \in\langle a\rangle \Phi(G)$, choose $b \in G \backslash\langle a\rangle \Phi(G)$. Then $b u \in G \backslash \Phi(G)$ and either $[b, a, a]$ or $[b u, a, a]$ is non trivial.

TheOREM 3. Let $G$ be a finite 3-generator p-group in $\mathcal{U}_{2,1}$ of class 3. Then there exist generators $\{a, b, c\}$ for $G$ such that a has maximal order in $G$, and $G$ has relations:

$$
\begin{aligned}
& {[b, a, a]=a^{3 p^{r}}, \quad[b, a, b]=b^{3 p^{r}}} \\
& {[c, a, b]=[b, c, a]=c^{p^{r}},} \\
& {[c, a, a]=[c, a, c]=[c, b, b]=[c, b, c]=1 .}
\end{aligned}
$$

Proof. Choose $a$ and $b$ in $G$ such that

(i) $a$ is of maximal order in $G$,

(ii) $\{a, b\}$ can be extended to a set of (non redundant) generators for $G$, and

(iii) $r_{a b}$ is minimal in $\cup_{a} \mathcal{R}(a)$ for $a$ of maximal order in $G$, and $b$ satisfying (ii).

Lemma 2 ensures that this choice is possible. Put $r=r_{a b}$. Then $[b, a, a]=a^{\alpha p^{r}}$ and $[b, a, b]=b^{\alpha p^{r}}$. Let $\{a, b, y\}$ be a set of generators for $G$. Since $G$ is in $\mathcal{U}_{2,1}$ and every 2-generator subgroup is in $\mathcal{W}_{2}$ we can assume that $G$ has the following relations;

$$
\begin{array}{ll}
{[b, a, a]=a^{\rho p^{r}},} & {[b, a, b]=b^{\rho p^{r}},} \\
{[y, a, a]=a^{\sigma p^{s}},} & {[y, a, y]=y^{\sigma p^{s}},} \\
{[y, b, b]=b^{\tau p^{t}},} & {[y, b, y]=y^{\tau p^{t}},}
\end{array}
$$

where $\rho, \sigma$ and $\tau$ are integers and $(\rho \sigma \tau, p)=1$, and $r, s$ and $t$ are positive integers. By the choice of $a$ and $b, s \geq r$. By taking suitable powers of $b$ and $y$ we may assume that $\sigma=\tau=1$. Since $G$ has class three, $a^{p^{r}}, b^{p^{r}}, a^{p^{s}}, y^{p^{s}}, b^{p^{t}}$ and $y^{p^{t}}$ are central. Since the group is regular and $r \leq s, 1=\left[y, a^{p^{r}}\right]=[y, a]^{p^{r}}=\left[y^{p^{r}}, a\right]$. Similarly $\left[y^{p^{r}}, b\right]=1$, $\left[a^{p^{t}}, y\right]=1$ and $\left[a^{p^{t}}, b\right]=1$. So if $m=\min \{r, t\}$, then $\left\{a^{p^{m}}, b^{p^{m}}, y^{p^{m}}\right\} \subseteq \zeta(G)$.

Assume that $[y, a, a] \neq 1$. If $[y, a, a]=1$, then $[y, a, y]=1$, making the following step unnecessary. Put $x=y^{\rho} b^{p^{s-r}}$. Then

$$
[x, a, a]=\left[y^{\rho}, a, a\right]\left[b^{p^{s-r}}, a, a\right]=a^{\rho p^{t}}\left(a^{\rho p^{r}}\right)^{-p^{t-r}}=1 .
$$

Since $|x| \leq|a|$, the regularity of the group gives $[x, a, x]=1$. Also $[x, b, b]=b^{\rho p^{t}}$ and $[x, b, x]=x^{\rho p^{t}}$. So $G=\langle a, b, x\rangle$ and has relations 


$$
\begin{aligned}
& {[b, a, a]=a^{\rho p^{r}}, \quad[b, a, b]=b^{\rho p^{r}},} \\
& {[x, b, b]=b^{\rho p^{t}}, \quad[x, b, x]=x^{\rho p^{t}},} \\
& {[x, a, a]=[x, a, x]=1 .}
\end{aligned}
$$

By taking a suitable power of $b$ we can adjust $\rho$. For convenience in the next step, choose $\rho=3$.

We get further information about $G$ by considering 2-generator subgroups of $G$. Each 2-generator subgroup of $G$ has Wielandt length two with the property that its commutator subgroup is in its Wielandt subgroup. In $\langle a, b x\rangle$,

$$
[a, b x, a]=[a, b, a][a, x, a]=a^{-3 p^{r}} .
$$

Hence $[a, b x, b x]=(b x)^{-3 p^{r}}=b^{-3 p^{r}} x^{-3 p^{r}}$. On expansion

$$
[a, b x, b x]=[a, b, b][a, b, x][a, x, b][a, x, x]=b^{-3 p^{r}}[a, b, x][a, x, b] .
$$

Put $w=[x, a, b]$. Then $[a, b, x]=w x^{-3 p^{r}}$, and from the Jacobi identity, $[x, b, a]=$ $w^{2} x^{-3 p^{r}}$. If $|b| \geq|x|$, consider $\langle b, a x\rangle$. Here

$$
[b, a x, b]=[b, a, b][b, x, b]=b^{3 p^{r}} b^{-3 p^{t}}=b^{3\left(p^{r}-p^{t}\right)} .
$$

From this, $[b, a x, g]=g^{3\left(p^{r}-p^{t}\right)-\lambda p^{l}}$ for $g \in\langle b, a x\rangle$, where $(\lambda, p)=1$ and $b^{p^{l}}=1$ and by the assumption on the orders of $b$ and $x, x^{p^{l}}=1$. Consequently,

$$
[b, a x, a x]=(a x)^{3\left(p^{r}-p^{t}\right)-\lambda p^{l}}=a^{3\left(p^{r}-p^{t}\right)} x^{3\left(p^{r}-p^{t}\right)} a^{-\lambda p^{l}} .
$$

Also

$$
\begin{aligned}
{[b, a x, a x] } & =[b, a, a][b, a, x][b, x, a][b, x, x] \\
& =a^{3 p^{r}} w^{-1} x^{3 p^{r}} w^{-2} x^{3 p^{r}} x^{-3 p^{t}} \\
& =a^{3 p^{r}} x^{3 p^{r}-3 p^{t}} w^{-3} x^{3 p^{r}}
\end{aligned}
$$

giving

$$
w^{3}=x^{3 p^{r}} a^{3 p^{t}} a^{\lambda p^{l}}
$$

If $a^{p^{l}}=1$, then $w=x^{p^{r}} a^{p^{t}}$. If $a^{p^{l}} \neq 1$, put $3 p^{t}+\lambda p^{l}=3 \bar{\tau} p^{\bar{t}}$. Then $[x, b, b]=b^{3 \bar{\tau} p^{\bar{t}}}$, $[x, b, x]=x^{3 \bar{\tau} p^{\bar{t}}}$, and $w=x^{p^{r}} y^{\bar{\tau} p^{\bar{t}}}$. By choosing a suitable power of $x$, we may assume $\bar{\tau}=1$. We have shown that the following relations hold in $G$ :

$$
\begin{aligned}
& {[b, a, a]=a^{3 p^{r}}, \quad[b, a, b]=b^{3 p^{r}},} \\
& {[x, b, b]=b^{3 p^{t}}, \quad[x, b, x]=x^{3 p^{t}},} \\
& {[x, a, b]=a^{p^{t}} x^{p^{r}}, \quad[b, x, a]=a^{-2 p^{t}} x^{p^{r}}} \\
& {[x, a, a]=[x, a, x]=1}
\end{aligned}
$$

where $t$ represents $t$ or $\bar{t}$, as necessary. The same result is achieved by a similar calculation if $|x|>|b|$. 
If $t<r$, put $a^{\prime}=a y^{p^{r-t}-1}$. Then $\left|a^{\prime}\right|=|a|$, and $\left[b, a^{\prime}, a^{\prime}\right]=\left(a^{\prime}\right)^{3 p^{t}},\left[b, a^{\prime}, b\right]=b^{3 p^{t}}$, contradicting the choice of $a$ and $b$. If $t \geq r$, put $c=x a^{p^{t-r}}$. Then $G$ has the required relations.

THeORem 4. Let $G$ be a finite p-group in $\mathcal{U}_{2,1}$. Then there exists a set of generators $\left\{a, b, x_{3}, \ldots, x_{n}\right\}$ for $G$, where $a$ is of maximal order in $G$, and the following relations are satisfied:

$$
\begin{aligned}
& {[b, a, a]=a^{3 p^{r}}, \quad[b, a, b]=b^{3 p^{r}}} \\
& {[x, a, b]=[b, x, a]=x^{p^{r}}} \\
& {[x, a, a]=[x, a, x]=[x, b, b]=[x, b, x]=1, \gamma_{4}(G)=1,}
\end{aligned}
$$

where $x \in\left\{x_{3}, \ldots, x_{n}\right\}$.

Proof. Choose $a$ and $b$ in $G$ such that

(i) $a$ is of maximal order in $G$,

(ii) $\{a, b\}$ can be extended to a set of (non redundant) generators for $G$, and

(iii) $r_{a b}$ is minimal in $\cup_{a} \mathcal{R}(a)$ for $a$ of maximal order in $G$, and $b$ satisfying (ii).

Put $r=r_{a b}$. Let $\left\{a, b, y_{3}, \ldots y_{n}\right\}$ be a set of generators for $G$. Put $H_{i}=\left\langle a, b, y_{i}\right\rangle$. As in Theorem 3 , each group $H_{i}$ has generators $\left\{a, b_{i}, x_{i}\right\}$ satisfying

$$
\begin{aligned}
& {\left[b_{i}, a, a\right]=a^{3 p^{r}}, \quad\left[b_{i}, a, b_{i}\right]=b_{i}^{3 p^{r}}} \\
& {\left[x_{i}, a, b_{i}\right]=\left[b_{i}, x_{i}, a\right]=x_{i}^{p^{r}}} \\
& {\left[x_{i}, a, a\right]=\left[x_{i}, a, x_{i}\right]=\left[x_{i}, b_{i}, b_{i}\right]=\left[x_{i}, b_{i}, x_{i}\right]=1, \gamma_{4}(G)=1 .}
\end{aligned}
$$

This almost completes the proof of the theorem, except that each $b_{i}$ is a (possibly different) power of the original element $b$. However, since each $b_{i}$ satisfies $\left[b_{i}, a, a\right]=a^{3 p^{r}}$ and $\left[b_{i}, a, b_{i}\right]=b_{i}^{3 p^{r}}$ and $b_{i}$ only differs from $b_{j}$ by a power of $b, b_{j}$ will also satisfy $\left[x_{i}, a, b_{j}\right]=\left[b_{j}, x_{i}, a\right]=x_{i}^{p^{r}}$. So any $b_{i}$ will be suitable to satisfy the relations given in the statement of the theorem. For convenience, choose $b=b_{3}$.

The statement of Theorem 4 does not yet give a presentation for a group in $\mathcal{U}_{2,1}$, but perhaps it can be thought of as a "partial presentation". The designation "partial presentation" is used for convenience and refers to the presentation of a group of which the group having the "partial presentation" is a quotient. Call this partial presentation $\mathcal{P}_{1}$. We define a series of partial presentations, $\mathcal{P}_{k}$, on the generating set $D_{k}$, where

$$
D_{k}=\left\{a_{1}, b_{1}, \ldots, a_{k}, b_{k}, x_{2 k+1}, \ldots, x_{n}\right\}
$$

and $a_{i}$ is of maximal order in $\left\langle a_{i}, b_{i}, \ldots, a_{k}, b_{k}, x_{2 k+1}, \ldots, x_{n}\right\rangle, 1 \leq i \leq k$. The partial presentation $\mathcal{P}_{1}$ is given by:

$$
\begin{aligned}
& {\left[b_{1}, a_{1}, a_{1}\right]=a_{1}^{3 p^{r_{1}}}, \quad\left[b_{1}, a_{1}, b_{1}\right]=b_{1}^{3 p^{r_{1}}},} \\
& {\left[x, a_{1}, b_{1}\right]=\left[b_{1}, x, a_{1}\right]=x^{p^{r_{1}}},} \\
& {\left[x, a_{1}, a_{1}\right]=\left[x, a_{1}, x\right]=\left[x, b_{1}, b_{1}\right]=\left[x, b_{1}, x\right]=1,}
\end{aligned}
$$


where $x \in D_{k} \backslash\left\{a_{1}, b_{1}\right\}$. The partial presentation $\mathcal{P}_{k}$ is given by:

$$
\begin{aligned}
& {\left[b_{i}, a_{i}, a_{i}\right]=a_{i}^{3 p^{r_{i}}}, \quad\left[b_{i}, a_{i}, b_{i}\right]=b_{i}^{3 p^{r_{i}}},} \\
& {\left[x, a_{i}, b_{i}\right]=\left[b_{i}, x, a_{i}\right]=x^{p^{r_{i}}},} \\
& {\left[x, a_{i}, a_{i}\right]=\left[x, a_{i}, x\right]=\left[x, b_{i}, b_{i}\right]=\left[x, b_{i}, x\right]=1,} \\
& {\left[y, a_{i}, a_{j}\right]=\left[y, a_{j}, a_{i}\right]=\left[y, b_{i}, b_{j}\right]=\left[y, b_{j}, b_{i}\right]=1,} \\
& {\left[y, a_{i}, b_{j}\right]=\left[y, b_{j}, a_{i}\right]=1,}
\end{aligned}
$$

where $i, j \in\{1, \ldots, k\}, i \neq j$, and $x \in D_{k}-\left\{a_{i}, b_{i}\right\}, y \in D_{k}-\left\{a_{i}, b_{i}, a_{j}, b_{j}\right\}$.

TheOREM 5. Let $G$ be a finite p-group of class three in $\mathcal{U}_{2,1}$ on $n$ generators, and let $k$ be a positive integer such that $2 k \leq n$. Then there exists a set of generators $D_{k}$ as given above, such that $G$ has a partial presentation $\mathcal{P}_{k}$.

The next series of lemmas is used in the proof of this theorem. In these lemmas the group $G$ has class three and is defined as follows:

$$
G=\left\langle a_{1}, b_{1}, \ldots, a_{k-1}, b_{k-1}, x_{2 k-1}, \ldots, x_{n}\right\rangle
$$

where the generators satisfy the relations $\mathcal{P}_{k-1}, 2 \leq 2 k \leq n$, and

$$
H_{i}=\left\langle a_{i}, b_{i}, \ldots, a_{k-1}, b_{k-1}, x_{2 k-1}, \ldots, x_{n}\right\rangle,
$$

and $a_{i}$ is of maximal order in $H_{i}, 1 \leq i \leq k-1$. Also

$$
H_{k}=\left\langle x_{2 k-1}, \ldots, x_{n}\right\rangle
$$

Lemma 6. Let a be an element of maximal order in the subgroup $H_{k}$ of $G$ and let $b \in H_{k}$ such that $[b, a, a]=a^{3 p^{t}}, \quad[b, a, b]=b^{3 p^{t}}$ for some integer $t$. Then there exists an integer $r$ such that

$$
[b, a, a]=a^{3 \rho p^{r}}, \quad[b, a, b]=b^{3 \rho p^{r}} \quad \text { and } \quad\left[a_{1}, a, b\right]=\left[b, a_{1}, a\right]=a^{\rho p^{r}} .
$$

Proof. Note that $\left[a_{1}, a b, a_{1}\right]=1$ which implies that

$$
1=\left[a_{1}, a b, a b\right]=\left[a_{1}, a, b\right]\left[a_{1}, b, a\right] .
$$

Put $w:=\left[a_{1}, a, b\right]=\left[b, a_{1}, a\right]$. By the Jacobi identity $w^{2}=\left[b, a, a_{1}\right]$. Further $\left[a, a_{1} b, a\right]=a^{-3 p^{t}}$ which implies

$$
\left[a, a_{1} b, a_{1} b\right]=\left(a_{1} b\right)^{-3 p^{t}-3 \lambda p^{l}}=a_{1}^{-3 p^{t}} b^{-3 p^{t}} a_{1}^{-3 \lambda p^{l}}
$$

where $(\lambda, p)=1$ and $a^{p^{l}}=1$. Also

$$
\begin{aligned}
{\left[a, a_{1} b, a_{1} b\right] } & =\left[a, a_{1}, b\right]\left[a, b, a_{1}\right][a, b, b] \\
& =w^{-3} b^{-3 p^{t}} .
\end{aligned}
$$


From this we get $w^{3}=a_{1}^{3 p^{t}+3 \lambda p^{l}}$. If $a^{p^{l}}=1$, put $r:=t$ and we have the required result. Otherwise, put $3 \rho p^{r}:=3 p^{t}+3 \lambda p^{l}$ which gives $w=a_{1}^{\rho p^{r}}$. Then $[b, a, a]=a^{3 \rho p^{r}}$ and $[b, a, b]=b^{3 \rho p^{r}}$.

Lemma 7. Let a be an element of maximal order in the subgroup $H_{k}$ of $G$ and let $b \in H_{k}$ such that $[b, a, a]=a^{3 p^{r}}, \quad[b, a, b]=b^{3 p^{r}}$. If also $\left[a_{1}, a, b\right]=\left[b, a_{1}, a\right]=a^{p^{r}}$, then $[u, a, b]=[b, u, a]=u^{p^{r}}$ for $u \in\left\{b_{1}, a_{2}, b_{2}, \ldots, a_{k-1}, b_{k-1}\right\}$.

Proof. If $|u|=\left|a_{1}\right|$, then $[u, a b, u]=1$ giving $1=[u, a b, a b]=[u, a, b][u, b, a]$. If $|u|<\left|a_{1}\right|$, then $\left|u a_{1}\right|=\left|a_{1}\right|$ and

$\left[u a_{1}, a b, u a_{1}\right]=[u, a, u]\left[u, a, a_{1}\right][u, b, u]\left[u, b, a_{1}\right]\left[a_{1}, a, u\right]\left[a_{1}, a, a_{1}\right]\left[a_{1}, b, u\right]\left[a_{1}, b, a_{1}\right]$.

If $u \neq b_{1}$ all these terms are trivial. If $u=b_{1}$, then

$$
\left[u, a, a_{1}\right]=a^{p^{r_{1}}}, \quad\left[a_{1}, a, u\right]=a^{-p^{r_{1}}}, \quad\left[u, b, a_{1}\right]=b^{p^{r_{1}}}, \quad\left[a_{1}, b, u\right]=b^{-p^{r_{1}}},
$$

and all other terms are trivial. In all cases $\left[u a_{1}, a b, u a_{1}\right]=1$. So

$$
\begin{aligned}
1=\left[u a_{1}, a b, a b\right] & =[u, a, b][u, b, a] a_{1}^{p^{r}} a_{1}^{-p^{r}} \\
& =[u, a, b][u, b, a] .
\end{aligned}
$$

Put $w:=[u, a, b]=[b, u, a]$ and $w^{2}=[b, a, u]$. If $|a|=\left|a_{1}\right|$, then $[a, u b, a]=a^{-3 p^{r}}$ implies

$$
(u b)^{-3 p^{r}}=[a, u b, u b]=w^{-3} b^{-3 p^{r}} .
$$

Thus $w=u^{p^{r}}$. If $|a|<\left|a_{1}\right|$, then $\left|a a_{1}\right|=\left|a_{1}\right|$ and

$$
\left[a a_{1}, u b, a a_{1}\right]= \begin{cases}\left(a a_{1}\right)^{-3 p^{r_{1}}-3 p^{r}} & \text { if } u=b_{1}, \\ \left(a a_{1}\right)^{-3 p^{r}} & \text { otherwise. }\end{cases}
$$

So, if $u=b_{1}$,

$$
\left(b_{1} b\right)^{-3 p^{r_{1}}-3 p^{r}}=\left[a a_{1}, b_{1} b, b_{1} b\right]=b_{1}^{-3 p^{r_{1}}} b^{-3 p^{r_{1}}-3 p^{r}} w^{-3},
$$

giving $w=b_{1}^{p^{r}}$. If $u \neq b_{1}$ a similar calculation gives $w=u^{p^{r}}$, as required.

Lemma 8. Let a be an element of maximal order in the subgroup $H_{k}$ of $G$ and let $b, x \in H_{k}$ such that $\left|\left[a_{1}, a, b\right]\right| \geq\left|\left[a_{1}, a, x\right]\right|,\left[a_{1}, a, b\right]=a_{1}^{p^{r}}$, and $[a, x, x]=[a, x, a]=1$. Then there exists $x^{\prime}=x b^{m}$ such that $\left[x^{\prime}, a, a_{1}\right]=\left[x^{\prime}, a_{1}, a\right]=1$.

Proof. The proof is similar to the previous proofs, and $\left[a_{1}, a x, a_{1}\right]=1$ implies $1=\left[a_{1}, a x, a x\right]=\left[a_{1}, x, a\right]\left[a_{1}, a, x\right]$. Put $w:=\left[a_{1}, a, x\right]=\left[x, a_{1}, a\right]$ and $w^{2}=\left[x, a, a_{1}\right]$. Also $\left[a, a_{1} x, a\right]=1$, but since $a$ is not necessarily of maximal order in $G$ we can only deduce that there exist integers $\lambda$ and $l \geq 1$ such that $(\lambda, p)=1, a^{p^{l}}=1$ and $\left[a, a_{1} x, a_{1} x\right]=\left(a_{1} x\right)^{-3 \lambda p^{l}}=a_{1}^{-3 \lambda p^{l}}$. Upon expansion $\left[a, a_{1} x, a_{1} x\right]=\left[a, a_{1}, x\right]\left[a, x, a_{1}\right]=$ $w^{-3}$, giving $w=a_{1}^{\lambda p^{l}}$. If $a_{1}^{p^{l}}=1$, then $w=1$ and we put $x^{\prime}=x$. Otherwise put $x^{\prime}=x b^{-\lambda p^{l-r}}$. The condition on the orders of $\left[a_{1}, a, b\right]$ and $\left[a_{1}, a, x\right]$ ensures that $l \geq r$. Then $\left[a_{1}, a, x^{\prime}\right]=\left[x^{\prime}, a_{1}, a\right]=1$, giving the required result. 
Lemma 9. Let a be an element of maximal order in the subgroup $H_{k}$ of $G$ and assume that $[z, a, b]=[b, z, a]=z^{p^{r}}$ for $z \in\left\{a_{1}, b_{1}, \ldots, a_{k-1}, b_{k-1}\right\}$. Let $x \in H_{k}$ such that $\left[x, a_{1}, a\right]=\left[x, a, a_{1}\right]=1$. Then $[u, a, x]=[u, x, a]=1$ for $u \in\left\{b_{1}, a_{2}, b_{2}, \ldots\right.$, $\left.a_{k-1}, b_{k-1}\right\}$.

Proof. Again, the proof is similar to previous proofs. If $|u|=\left|a_{1}\right|$, then $[u, a x, u]=1$ implies $1=[u, a x, a x]=[u, a, x][u, x, a]$. If $|u|<\left|a_{1}\right|$, then $\left|a_{1} u\right|=\left|a_{1}\right|$, together with $\left[a_{1} u, a x, a_{1} u\right]=1$, implies $1=\left[a_{1} u, a x, a x\right]=[u, a, x][u, x, a]$. Set $w:=[u, a, x]=[x, u, a]$ and $w^{2}=[x, a, u]$. If $|a|=\left|a_{1}\right|$, then $[a, u x, a]=1$ implies $1=[a, u x, u x]=[a, u, x][a, x, u]=w^{-3}$, giving $w=1$, as required. If $|a|<\left|a_{1}\right|$, then $\left|a a_{1}\right|=\left|a_{1}\right|$. In this case

$$
\left[a a_{1}, u x, a a_{1}\right]= \begin{cases}\left(a a_{1}\right)^{-3 p^{r}} & \text { if } u=b_{1} \\ 1 & \text { otherwise }\end{cases}
$$

Hence

$$
\left[a a_{1}, u x, u x\right]= \begin{cases}(u x)^{-3 p^{r}} & \text { if } u=b_{1} \\ 1 & \text { otherwise }\end{cases}
$$

In either case, upon expansion of the commutators, we get $w^{3}=1$, which implies $w=1$, completing the proof.

Proof of Theorem 5. The partial presentation $\mathcal{P}_{1}$ is given by Theorem 4. Assume that the group $G$ has partial presentation $\mathcal{P}_{k-1}, 2<k \leq 2 n$. We prove the theorem by deriving the presentation $\mathcal{P}_{k}$. Let $H_{k}=\left\langle x_{2 k-1}, \ldots, x_{n}\right\rangle$. Assume that $H_{k}$ has class three. By Theorem 4 there exist generators $\left\{a, b, z_{2 k+1}, \ldots, z_{n}\right\}$ for $H_{k}$ with the following properties:

$$
\begin{aligned}
& {[b, a, a]=a^{3 p^{t}}, \quad[b, a, b]=b^{3 p^{t}}} \\
& {[z, a, b]=[b, z, a]=z^{p^{t}}} \\
& {[z, a, a]=[z, a, z]=[z, b, b]=[z, b, z]=1, \gamma_{4}\left(H_{k}\right)=1,}
\end{aligned}
$$

where $\langle a\rangle \cap\langle b\rangle=1$, and $z \in\left\{z_{2 k+1} \ldots, z_{n}\right\}$. Since

$$
\left[x, a_{i}, b_{i}\right]=\left[b_{i}, x, a_{i}\right]=x^{p^{r_{i}}} \quad \text { and } \quad\left[x, a_{i}, a_{i}\right]=\left[x, a_{i}, x\right]=\left[x, b_{i}, b_{i}\right]=\left[x, b_{i}, x\right]=1
$$

for $i \in\{1, \ldots, k\}$ and $x \in D_{k}-\left\{a_{i}, b_{i}\right\}$, for each $i$ these relations are also true for $x \in\left\langle D_{k}-\left\{a_{i}, b_{i}\right\}\right\rangle$. In particular,

$$
\left[v, a_{i}, b_{i}\right]=\left[b_{i}, v, a_{i}\right]=v^{p^{r_{i}}} \quad \text { and } \quad\left[v, a_{i}, a_{i}\right]=\left[v, a_{i}, v\right]=\left[v, b_{i}, b_{i}\right]=\left[v, b_{i}, v\right]=1
$$

for $v \in\left\{a, b, z_{2 k+1} \ldots, z_{n}\right\}$.

To prove the theorem we need to define $r_{k}$ and then show that

$$
[u, a, b]=[b, u, a]=u^{p^{r} k}
$$

for $u \in\left\{a_{1}, b_{1}, \ldots, a_{k-1}, b_{k-1}\right\}$. Then we need to show that

$$
[z, a, u]=[z, u, a]=[z, b, u]=[z, u, b]=1
$$


for $z \in\left\{z_{2 k+1} \ldots, z_{n}\right\}$ (or an appropriate set of generators) and $u$ as above. Lemma 6 provides an integer, call it $r_{k}$, such that

$$
[b, a, a]=a^{3 \rho p^{r_{k}}}, \quad[b, a, b]=b^{3 \rho p^{r_{k}}} \quad \text { and } \quad\left[a_{1}, a, b\right]=\left[b, a_{1}, a\right]=a^{\rho p^{r_{k}}},
$$

where $\rho p^{r_{k}}:=p^{t}+\lambda p^{l},(\lambda, p)=1$ and $a^{p^{l}}=1$. Since $a$ has maximal order in $H_{k}$, we also have that $[z, a, b]=[b, z, a]=z^{\rho p^{r_{k}}}$ for $z \in\left\{z_{2 k+1} \ldots, z_{n}\right\}$. By choosing a suitable power of $b$ we can assume that $\rho=1$. Lemma 7 now completes the proof of (10).

Let $z \in\left\{z_{2 k+1} \ldots, z_{n}\right\}$. If $\left[a_{1}, a, z\right]=\left[a_{1}, z, a\right]=1$, put $z^{\prime}:=z$. Otherwise, from the proof of Lemma $8,\left[a_{1}, a, z\right]=a_{1}^{\lambda p^{l}}$, where $a^{p^{l}}=1$. Since $[b, a, a]=a^{p^{p_{k}}} \neq 1$, this ensures that $l \geq r_{k}$ and $\left|\left[a_{1}, a, b\right]\right| \geq\left|\left[a_{1}, a, z\right]\right|$. In this case, replace $z$ by $z^{\prime}=z b^{\lambda p^{l}}$. Then $\left\{a, b, z_{2 k+1}^{\prime}, \ldots, z_{n}^{\prime}\right\}$ generates $H_{k}$, and $\left[z, a, a_{1}\right]=\left[z, a_{1}, a\right]=1$ for $z \in\left\{z_{2 k+1}^{\prime}, \ldots, z_{n}^{\prime}\right\}$. It is also true that $\left[z, a_{i}, b_{i}\right]=\left[b_{i}, z, a_{i}\right]=z^{p^{r_{i}}}$, and $\left[z, a_{i}, a_{i}\right]=\left[z, a_{i}, z\right]=\left[z, b_{i}, b_{i}\right]=\left[z, b_{i}, z\right]=1,1 \leq i \leq k-1$. Lemma 9 now completes the first part of (11), namely, that $[z, a, u]=[z, u, a]=$ for $z \in\left\{z_{2 k+1}^{\prime}, \ldots, z_{n}^{\prime}\right\}$ and $u \in\left\{a_{1}, b_{1}, \ldots, a_{k-1}, b_{k-1}\right\}$.

To complete the proof of (11) we first consider $\left[z, a_{1}, b\right]$. If $|b|=|a|$ we can again use Lemma 8, with a small modification, to show that $\left[z^{\prime}, a_{1}, b\right]=\left[z^{\prime}, b, a_{1}\right]=1$, where $z^{\prime}=z a^{m}$, for some integer $m$ and $z \in\left\{z_{2 k+1}^{\prime}, \ldots, z_{n}^{\prime}\right\}$. If $|b|<|a|$, we use Lemma 8 also, for $a^{\prime}=a b$ noting that $\left[a_{1}, a^{\prime}, a\right]=a_{1}^{-p^{r} k}$. With a slight modification to the proof of Lemma 8 , we again find $z^{\prime}=z a^{m}$ for some integer $m$ such that for each $z^{\prime},\left[z^{\prime}, a_{1}, a^{\prime}\right]=\left[z^{\prime}, a^{\prime}, a_{1}\right]=1$. Since $\left[z^{\prime}, a_{1}, a\right]=\left[z, a_{1}, a\right]\left[a^{m}, a_{1}, a\right]=1$ and $\left[z^{\prime}, a, a_{1}\right]=1$, this is enough to show that $\left[z^{\prime}, a_{1}, b\right]=\left[z^{\prime}, b, a_{1}\right]=1$. Again the relations already established for $z \in\left\{z_{2 k+1}^{\prime}, \ldots, z_{n}^{\prime}\right\}$ also hold for $z \in\left\{z_{2 k+1}^{\prime \prime}, \ldots, z_{n}^{\prime \prime}\right\}$, and $H_{k}=\left\langle a, b, z_{2 k+1}^{\prime \prime}, \ldots, z_{n}^{\prime \prime}\right\rangle$. Lemma 9 now completes the proof of (11).

We consider the situation when $H_{k}$ has class two. In this case choose $a$ so that $a$ has maximal order in $H_{k}$, and choose $b$ so that $b \in H_{k} \backslash \Phi\left(H_{k}\right)$ and $\left|\left[a_{1}, a, b\right]\right| \geq\left|\left[a_{1}, a, x\right]\right|$ for any $x \in H_{k} \backslash \Phi\left(H_{k}\right)$. If $\left[a_{1}, a, x\right]=1$ for all $x \in H_{k}$, the choice of $b$ is arbitrary. Choose $\left\{z_{2 k+1} \ldots, z_{n}\right\}$ so that $\left\{a, b, z_{2 k+1} \ldots, z_{n}\right\}$ is a generating set for $H_{k}$. With the convention that $[b, a, a]=a^{3 p^{t}}$ for some integer $t \geq|a|$, the proof follows as for the case when $H_{k}$ has class three.

If we now put $a_{k}:=a, b_{k}:=b$ and $u_{i}:=z_{i}^{\prime \prime}, 2 k+1 \leq i \leq n$, then the generators $\left\{a_{1}, b_{1}, \ldots, a_{k}, b_{k}, u_{2 k+1}, \ldots, u_{n}\right\}$ for $G$ satisfy the relations $\mathcal{P}_{k}$.

Proof of Theorem A. If $G$ has class less than three, the theorem is true. So assume that $G$ has class three and is a group on $m$ generators. If $m$ is even put $n=m / 2$. Then by Theorem $4 G$ has the partial presentation $P_{n}$. This is the required presentation, except that the integers $r_{i}$ might not be ordered as stated in the Theorem. By changing the labelling of the generators we can ensure that $r_{1} \leq r_{2} \leq \cdots \leq r_{n}$.

If $m$ is odd, put $n=(m+1) / 2$. Then from Theorem $4 G$ has the partial presentation $P_{n-1}$, on generators $D_{n-1}=\left\{a_{1}, b_{1}, \ldots, a_{n-1}, b_{n-1}, x_{m}\right\}$. Again, by changing the labelling of the generators, we can ensure that $r_{1} \leq \cdots \leq r_{n-1}$.Put $a_{n}=x_{m}$ and $r_{n}=\exp (G)+1$. Then $G$ is a homomorphic image of $G\left(r_{1}, \ldots, r_{n}\right)$.

\section{REFERENCES}

1. C. Cooper, Power automorphisms of a group, Math. Z. 107 (1968), 335-356. 
2. P. Hall, A contribution to the theory of groups of prime power order, Proc. London Math. Soc. (2) 36 (1933), 29-95.

3. S. K. Mahdavianary, A classification of 2-generator $p$-groups, $p \geq 3$, with many subgroups 2-subnormal, Arch. Math. (Basel) 43 (1987), 97-107.

4. E. A. Ormerod, Groups of Wielandt length two, Math. Proc. Camb. Phil. Soc. 110 (1991), 229-244.

5. G. Parmeggiani, On finite $p$-groups of odd order with many subgroups 2-subnormal, Comm. Algebra 24 (1996), 2707-2719.

6. M. Stadelmann, Gruppen mit vielen Subnormalteilern vom Defekt zwei (Dissertation, Würzburg, 1976). 